\title{
Vascularized Bipedicled Pericranial Flaps for Reconstruction of Chronic Scalp Ulcer Occurring after Cranioplasty
}

\author{
Seok Ho Yoon, Jin Sik Burm, Won Yong Yang, Sang Yoon Kang \\ Department of Plastic and Reconstructive Surgery, Kyung Hee University School of Medicine, Seoul, Korea
}

Background Intractable chronic scalp ulcers with cranial bone exposure can occur along the incision after cranioplasty, posing challenges for clinicians. They occur as a result of severe scarring, poor blood circulation of the scalp, and focal osteomyelitis. We successfully repaired these scalp ulcers using a vascularized bipedicled pericranial flap after complete debridement. Methods Six patients who underwent cranioplasty had chronic ulcers where the cranial bone, with or without the metal plate, was exposed along the incision line. After completely excising the ulcer and the adjacent scar tissue, subgaleal dissection was performed. We removed the osteomyelitic calvarial bone, the exposed metal plate, and granulation tissue. A bipedicled pericranial flap was elevated to cover the defect between the bone graft or prosthesis and the normal cranial bone. It was transposed to the defect site and fixed using an absorbable suture. Scalp flaps were bilaterally advanced after relaxation incisions on the galea, and were closed without tension.

Results All the surgical wounds were completely healed with an improved aesthetic outcome, and there were no notable complications during a mean follow-up period of seven months.

Conclusions A bipedicled pericranial flap is vascularized, prompting wound healing without donor site morbidity. This may be an effective modality for treating chronic scalp ulcer accompanied by the exposure of the cranial bone after cranioplasty.

Keywords Surgical Flap / Scalp / Chronic disease
Correspondence: Jin Sik Burm Department of Plastic and Reconstructive Surgery, Kyung Hee University School of Medicine, 23 Kyungheedae-ro, Dongdaemun-gu, Seoul 130-872, Korea

Tel: +82-2-958-8431

Fax: $+82-2-963-5638$

E-mail: jsburm@gmail.com
This article was presented at the 70th Congress of the Korean Society of Plastic and Reconstructive Surgeons on November 9, 2012 in Seoul, Korea.

No potential conflict of interest relevant to this article was reported.

\section{INTRODUCTION}

In cases of calvarial bone defects occurring after surgery for head trauma, brain tumors, or strokes, defect sites are vulnerable to external impacts, causing aesthetic issues. Cranioplasty is widely performed using an autologous bone graft or bone prosthesis. A chronic ulcer is one of the complications that can occur following cranioplasty; it begins along the incision line and is a small lesion, accompanied by the exposure of bony structures or the bone-plate fixation system. A long-standing chronic ulcer poses a therapeutic challenge for clinicians. Small scalp defects are usually reconstructed using the primary closure or a local scalp flap, but in cases of chronic ulcers occurring after cranioplasty, we have sometimes experienced ulcer recurrence using these methods. Around these ulcers, the scalp is thin and there can be chronic inflammation at the surgical scar as well as

Copyright () 2013 The Korean Society of Plastic and Reconstructive Surgeons

This is an Open Access article distributed under the terms of the Creative Commons Attribution Non-Commercial License (http://creativecommons.org/

licenses/by-nc/3.0/) which permits unrestricted non-commercial use, distribution, and reproduction in any medium, provided the original work is properly cited.

www.e-aps.org 
wide subgalea scarring due to the previous surgery, which might not provide sufficient blood supply for wound healing and can make surgical wound closure difficult.

We hypothesized that the recurrence of chronic scalp ulcers is mainly due to poor vascularity in the scalp adjacent to the wound and the focal osteomyelitis. Thus, surgical outcomes might be improved by covering the defect site with a wellvascularized soft tissue flap. Pericranial flaps have a stable blood supply that includes an axial blood supply and a lack of donor deformity. Unipedicled pericranial flaps are vascularized, versatile flaps used for reconstruction of the skull base, frontal sinus, and frontonasal region $[1,2]$. However, the distal portion of unipedicled pericranial flaps does not have an axial blood supply, and there is no connection with the contralateral pericranial vessels crossing the cranial midline [3]. Therefore, we designed bipedicled pericranial flaps that provide a sufficient blood supply to the area afflicted by the chronic ulcer and obtained satisfactory outcomes. We describe our successful method and discuss the availability of the vascularized bipedicled pericranial flap for this difficult scalp reconstruction.

\section{METHODS}

\section{Patients}

The current study was conducted in six patients with chronic scalp ulcers where the bony structures were exposed following cranioplasty. Our clinical series of patients consisted of three men and three women, with a mean age of 49 years, all of whom underwent surgery more than twice through the same incision sites. The materials for cranioplasty were cryopreserved autogenous cranial bone in three patients and methylmethacrylate in three. After cranioplasty, the chronic scalp ulcer did not heal despite being treated with conventional treatments for up to 12 weeks. Its width varied, ranging from 5 to $12 \mathrm{~mm}$ (mean, 8.7 $\mathrm{mm}$ ) (Table 1). All the patients presented with an exposure of the bony structures, accompanied by focal osteomyelitis. The titanium plate was exposed in three patients, and all of the pa- tients demonstrated a thinned scalp around the ulcer and poor vascularity due to severe scarring. At the defect sites, cultures revealed the presence of such bacterial strains as Staphylococcus aureus, methicillin-resistant Staphylococcus aureus, Staphylococcus epidermidis, and Pseudomonas aeruginosa. The hospitalized patients received local wound management and systemic antibiotic administration; if applicable, they also underwent debridement and local flap surgery. The outpatients carried out wound management on their own, but in neither type of case did the wound recover despite all conventional treatments.

\section{Surgical technique}

At least a week before the operation, the hair and crust around the ulcer were removed for all of the patients. A bacterial culture test was performed at the margin of the ulcer, followed by systemic administration of sensitive antibiotics. The wound site was washed with normal saline at 12-hour intervals and for local antibacterial therapy, a gauze pad soaked in $10 \%$ povidone iodine was applied to the wound site; two days before surgery, it was replaced with saline-moistened gauze dressing.

The surgical procedures were performed in the supine position, prone position, or lateral decubitus position, depending on the location of the ulcer and previous incision. The wounds, including the ulcer, adjacent granulation tissue, thinned scalp, and previous scar were completely resected along the previous incision line. When there were any findings suggestive of osteomyelitis in the calvarial bone, the bone was shaved using a diamond burr. If possible, we also removed any exposed titanium plates. In patients where dead space with granulation tissue covering the dura mater was present below the bone graft, we also removed all the involved areas after full thickness bone shaving. We elevated the scalp flap bilaterally through the subgaleal plane, immediately under the galea aponeurotica, to ensure that enough subgaleal tissue would attach to the pericranium. At the non-craniectomy side, we designed a bipedicled pericranial flap, as wide as possible, to cover the defect site and the adjacent cranial bone. The designed flap obtained sufficient vascular-

\section{Table 1. Clinical characteristics of patients with chronic scalp ulcer after cranioplasty}

\begin{tabular}{|lccccc}
\hline Case & Sex/Age (yr) & Reasons for craniectomy & Graft materials & Time interval (wk) & $\begin{array}{c}\text { Ulcer size } \\
\text { (length } \times \text { width, mm) }\end{array}$ \\
\hline 1 & $\mathrm{~F} / 75$ & SAH & Cryo-preserved & 12 & $12 \times 10$ \\
2 & $\mathrm{~F} / 56$ & $\mathrm{SDH}$ & MMA & 24 & $5 \times 5,5 \times 5,5 \times 5$ \\
3 & $\mathrm{~S} / 25$ & $\mathrm{SDH}$ & Cryo-preserved & 16 & $20 \times 10$ \\
4 & $\mathrm{SDH}$ & MMA & 19 & $10 \times 7$ \\
5 & $\mathrm{~F} / 37$ & $\mathrm{SDH}, \mathrm{EDH}$ & MMA & 12 & $23 \times 12$ \\
6 & $\mathrm{SAH}$ & Cryo-preserved & 13 & $10 \times 8$ \\
\hline SAH, subarachnoid hemorrhage; SDH, subdural hemorrhage; MMA, methylmethacrylate; Cryo-preserved, Cryo-preserved autogenous cranial bone; EDH, epidural hemorrhage.
\end{tabular}


ity through the bilateral periosteal arteries. Then, we elevated the flap and transposed it to the defect site. To ensure that the pericranial flap did not bear tension, which can affect circulation, it was carefully sutured in place using an absorbable suture. When the bone defect was too large, we elevated an additional unipedicled pericranial flap from the craniectomy side, and then applied it to the defect sites to augment the depression. For approximation of the scalp flap, 2-3 relaxation incisions were made bilaterally on the galea aponeurotica parallel to the flap margin. This procedure increased the mobility of the flaps. After suturing the galea aponeurotica, the skin was closed using a tension free technique. When the scalp flap could not be closed due to severe scarring, we performed a split-thickness skin graft on the bipedicled pericranial flap.

\section{RESULTS}

All six patients had satisfactory outcomes with no postoperative complications, such as wound infection, dehiscence, or recurrence of ulcers, at a mean follow-up of seven months (range, 2 to 11 months). For the pedicle of the bipedicled pericranial flap, the occipital artery and the supraorbital artery (3 cases), the posterior auricular artery and the superficial temporal artery $(2$ cases), or the occipital artery and the superficial temporal artery ( 1 case) were used. In one case, there was a large bone defect, and for soft tissue augmentation we also used a unipedicled pericranial flap, where the superficial temporal artery served as a pedicle. In six patients, we performed a bilateral advancement flap for scalp closure and, in one patient, a split-thickness skin graft on the pericranial flap (Table 2).

\section{Case 1}

A 75-year-old woman was diagnosed with subarachnoid hemorrhage and a neurosurgeon performed aneurysmal clipping through a unicoronal incision. Two months later, the patient underwent cranioplasty using the cryopreserved autogenous cranial bone. At the sites of the surgical incision, there was a yellowish pus-like discharge and S. epidermidis was identified. Three weeks after cranioplasty, the wound was closed following incision and drainage. Due to tension at the suture sites, however, ischemic necrosis of the flap margin occurred. Conservative management did not improve wound healing. On physical examination, the patient had full-thickness skin defects $12 \mathrm{~mm} \times 10 \mathrm{~mm}$ in size on the scalp, accompanied by focal osteomyelitis (Fig. 1). Twelve weeks after cranioplasty, under local anesthesia, we removed all the dirty granulation tissues located in the dead space under the grafted cranial bone, after bone shaving. One week after debridement, under general anesthesia, we reconstructed the chronic ulcer using a bipedicled pericranial flap where the posterior auricular artery and the left superficial temporal artery served as a pedicle. The patient had a satisfactory treatment outcome, without any notable problems during an 11-month postoperative follow-up period.

\section{Case 2}

A 56-year-old woman was diagnosed with a subdural hemorrhage. The patient underwent decompression through an Sshaped incision at the parietal region. When her symptoms improved, the patient underwent cranioplasty using methylmethacrylate. Six months after cranioplasty, the patient presented to our clinic with multiple ulcers $5 \mathrm{~mm} \times 5 \mathrm{~mm}$ in size, where both the skull and the bone-plate fixation system were exposed along the incision line (Fig. 2). The scalp was also very thin along the incision line. A bacterial culture test was performed at the margin of the ulcer, which identified S. aureus and S. epidermidis.

After seven days of preoperative treatment, the thinned skin, the ulcer, the adjacent inflammatory tissue, and the metal plate were removed under general anesthesia. We shaved off some discolored bony structures with osteomyelitic changes, including the outer table of the calvarial bone and bone prosthesis. Then we used a bipedicled pericranial flap where the left occipital artery and the right superficial temporal artery served as pedicles. The surgery was successful without any notable complications during a six-month postoperative follow-up period.

\section{Table 2. Postoperative outcomes of reconstruction using a bipedicled pericranial flap}

\begin{tabular}{|c|c|c|c|c|}
\hline Case & Pedicle & Coverage & Follow-up period (mo) & Complication \\
\hline 1 & Rt. PAA \& Lt. STA & Local flap & 11 & - \\
\hline 2 & Both OA \& Rt. STA & Local flap & 6 & - \\
\hline 3 & Lt. OA \& Rt. SOA/Lt. STA ${ }^{\text {a) }}$ & Local flap & 4 & - \\
\hline 4 & Rt. PAA \& Lt. STA & Local flap & 8 & - \\
\hline 5 & Lt. OA \& Rt. SOA & Local flap & 2 & - \\
\hline 6 & Rt. OA \& Lt. SOA & Local flap & 9 & - \\
\hline
\end{tabular}



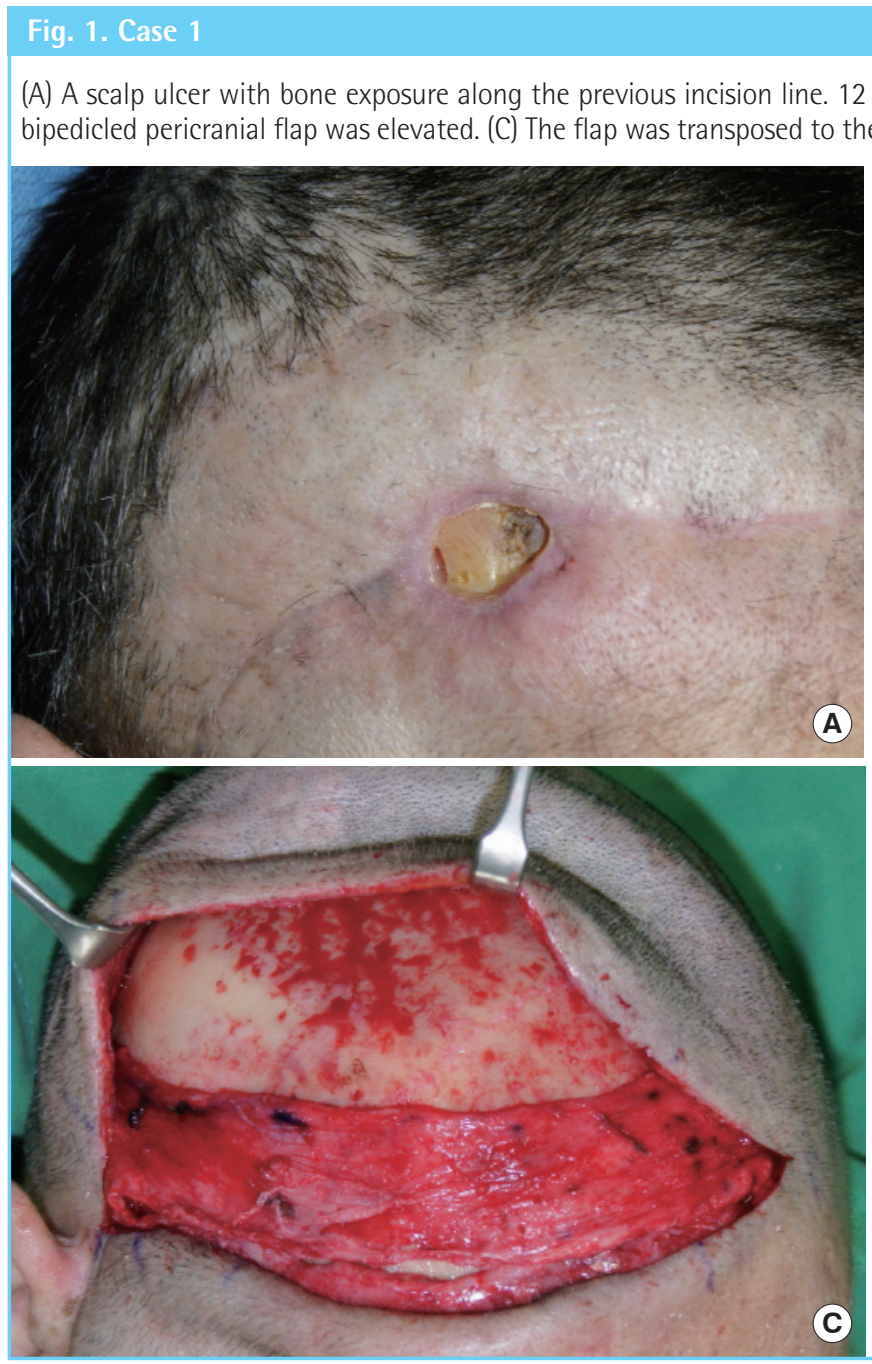

\section{Case 3}

A 25-year-old man was diagnosed with subdural hemorrhage and underwent decompression via a pterional approach by a neurosurgeon. When his symptoms improved, the patient underwent cranioplasty using the cryopreserved autogenous cranial bone. The ulcer occurred along the incision line. Despite being treated with ambulatory treatments, it had still not healed at postoperative four months. At the time of the outpatient visit, the patient presented with full-thickness scalp defects 20 $\mathrm{mm} \times 10 \mathrm{~mm}$ in size, where the discolored bone was exposed. In addition, the hair and follicles were also exposed in the subcutaneous layer adjacent to the ulcer (Fig. 3). After identifying methicillin-resistant Staphylococcus aureus, the patient received nine days of pre-treatment. Following the resection of the ulcer and the adjacent scar tissue, there was a discoloration in the overall layer of the grafted calvarial bone. We removed this layer, including its inner table. By using the left occipital artery, the right supraocular artery, and the supratrochlear artery as a pedicle, we performed a bipedicled pericranial flap. In addition,
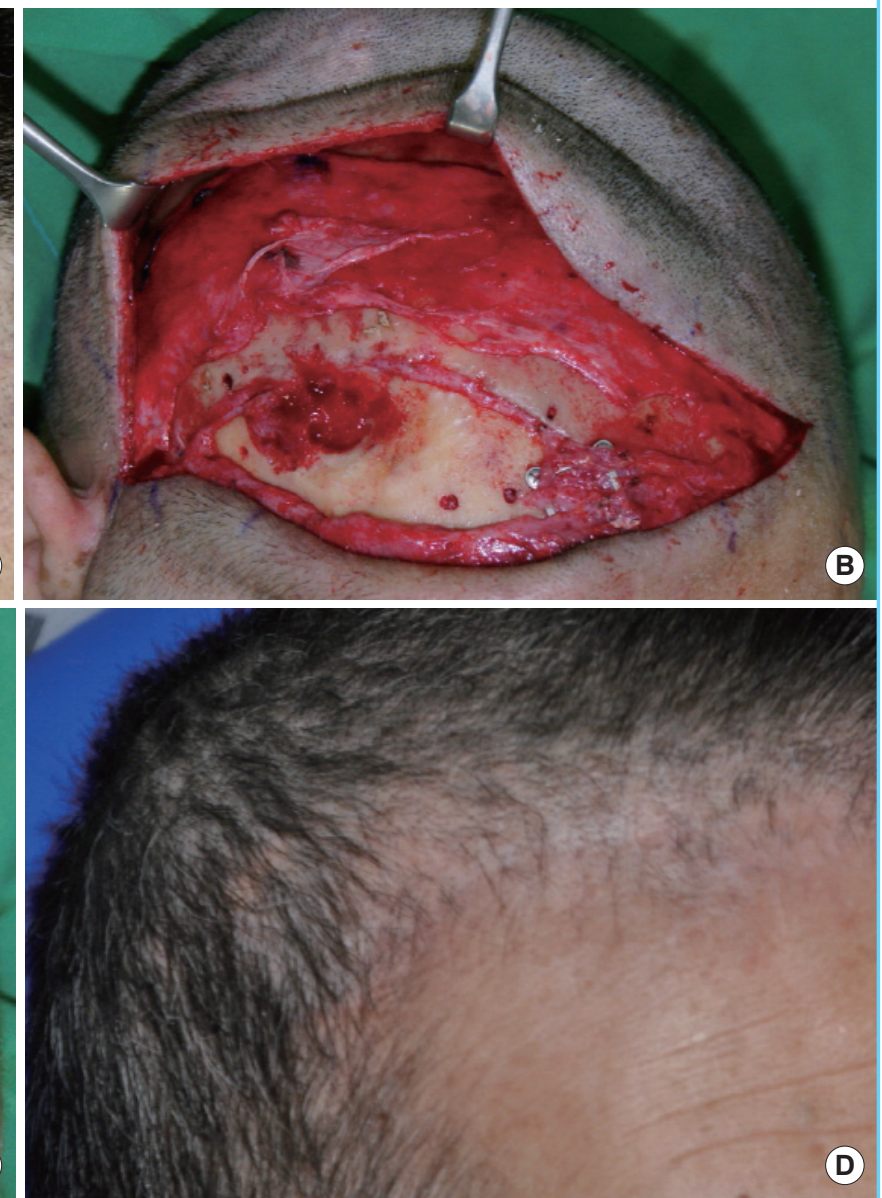

we elevated a unipedicled pericranial flap, where the superficial temporal artery served as a pedicle, to cover the defects after the removal of the cranial bone. Until postoperative four months, the patient had good aesthetic outcomes without developing any notable complications.

\section{DISCUSSION}

Intractable chronic scalp ulcers with cranial bone exposure can occur along the incision after cranioplasty. They can result from severe scarring, poor blood circulation within the scalp, or focal osteomyelitis. Therefore, the treatment goal is to remove all infectious material and to replace the infected tissue with a highdensity vascular network. Once the blood supply is improved, macrophages and leukocytes can come into contact with the wound bed, promoting phagocytosis, and this may stimulate wound healing [4].

We performed a complete resection of the granulation tissue and the thinned scalp around the ulcer, cutting along the incision 


\section{Fig. 2. Case 2}

(A) Multiple small scalp ulcers with exposed bone and metal plate. 6 months after cranioplasty. (B) After a total excision of the ulcer and inflammatory tissue, all the metal plates were removed. (C) A bipedicled pericranial flap was transposed to the left to cover the defect site. (D) View at postoperative 6 months.
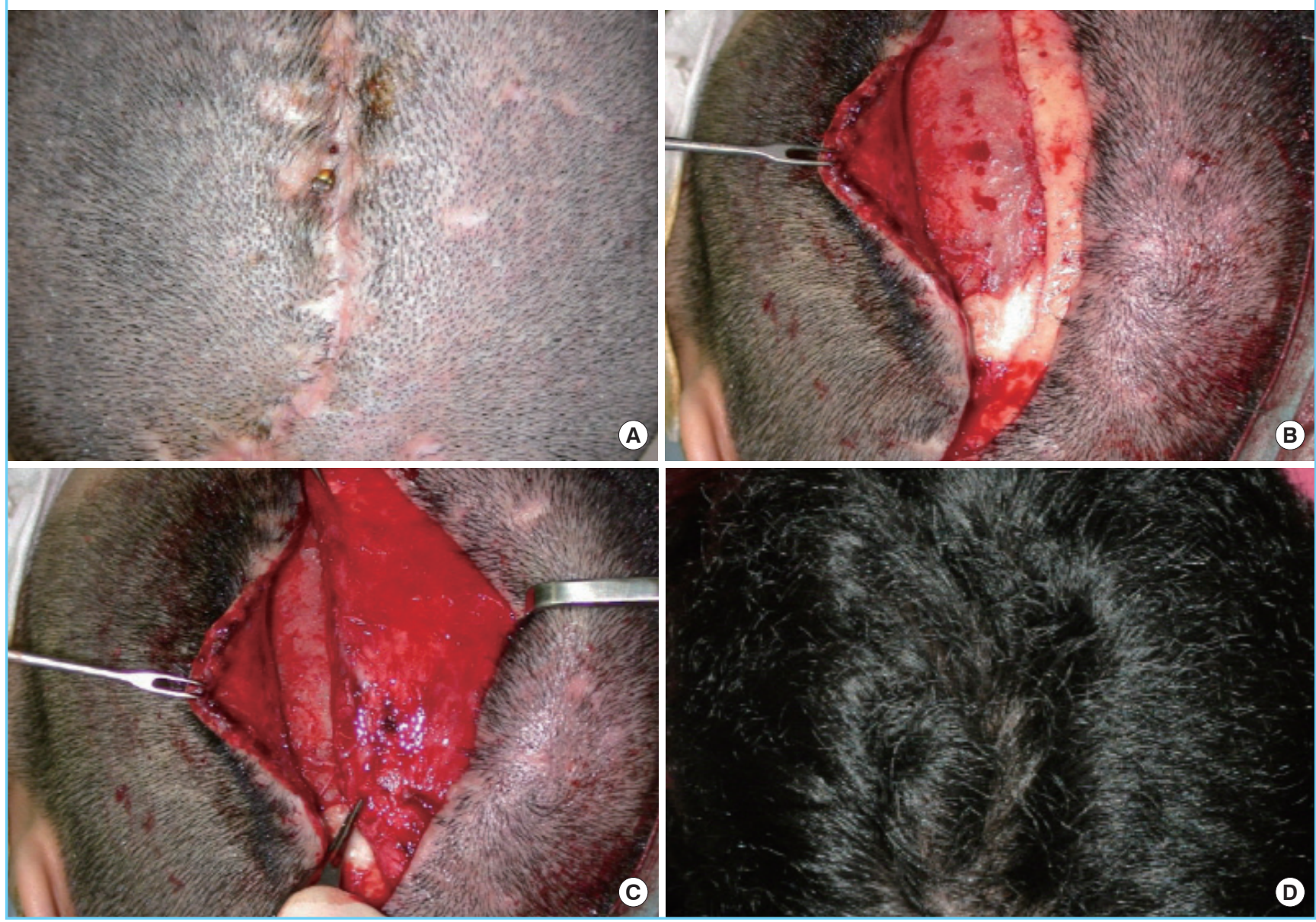

line. We also removed the bone-plate fixation system and the calvarial bone with chronic osteomyelitic changes in the relevant cases. In patients with progression of osteomyelitis, only the outer table of the calvarial bone was shaved, using a burr, until viable bone was encountered. In cases 1 and 3 , however, the osteomyelitis occurred in all of the layers of the calvarial bone. Therefore, we removed it, including its inner table. Moreover, in patients who had dead space or granulation tissue under the autologous bone graft or bone prosthesis, we removed the granulation tissue completely after the bone shaving. We shaved the margin of the bone defects along the oblique line to ensure that no angles were formed (Fig. 1B). Thus, we attempted to avoid causing dead space, covering the bone defects using a pericranial flap.

Scalp defects of moderate size $\left(2-25 \mathrm{~cm}^{2}\right)$ can be treated with a local flap in reconstruction [5], but if repetitive surgeries are performed, the mobility of flaps becomes poor because scarring occurs in the scalp areas. In addition, the flap design is difficult because surgical incisions have been made previously, which limits the availability of local flaps. A free flap can be used to reconstruct scalp defects where there is a concurrent presence of osteomyelitis $[4,6,7]$, but it is disadvantageous, as the operation time is long and the donor morbidity is relatively high. Pericranial flap is a composite flap involving the periosteum of the skull with subgaleal fascia. Their advantages include a convenient elevation for surgical procedures, versatility, and a lack of functional and morphological complications at donor sites. In addition, they receive blood supply from major arterial branches of the scalp in an axial pattern and from the occasional perforators arising from the calvarium. This ensures that a pericranial flap receives sufficient blood supply $[1,8,9]$.

The distal portion of unipedicled pericranial flaps does not have an axial blood supply, and there is no connection with the contralateral pericranial vessels crossing the cranial midline [3]. To maximize the vascularity of the flap, we designed a bilateral pericranial flap with the major arterial branches arising in the scalp, depending on the location of the ulcer (Fig. 4). The flap 


\section{Fig. 3. Case 3}

(A) A chronic ulcer and scalp contraction along the incision line. 4 months after cranioplasty. (B) A bipedicled pericranial flap (black arrows) and an additional unipedicled flap $(*)$ were transposed to the defect area. (C) Postoperative view at 11 days with a good scalp contour. (D) Postoperative view at 4 months.
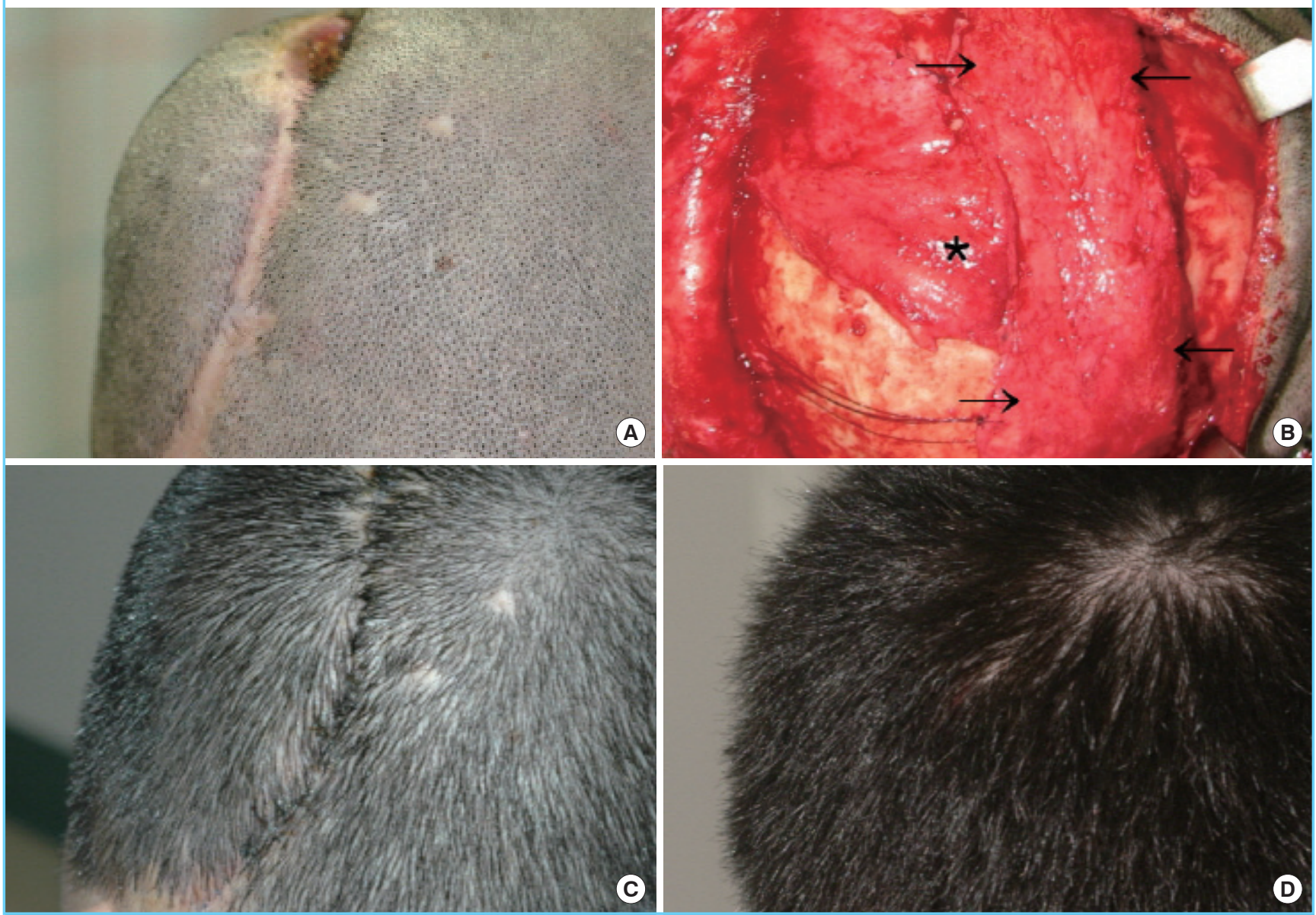

should be designed to be as wide as possible because cranial soft tissue shrinks when released owing to its elasticity [9]. In our experience, the width of the flap decreased from $7 \mathrm{~cm}$ at the time of design to less than $5 \mathrm{~cm}$ after flap elevation. If bipedicled pericranial flaps are elevated with sufficient dissection, they can be transposed to the defect site easily without back cutting due to a lack of mechanical strength. A back-cut into the pedicle may worsen the axial blood supply of the flap.

Because the pericranial flaps contain both the periosteum and subgaleal fascia, they are somewhat thick $[3,8]$. Thus, the flap is also a source of soft-tissue augmentation as a multi-layered flap, which increases bulk [2]. We performed a subgaleal dissection immediately under the galea aponeurotica and thereby maximized the volume of the flap, achieving a restoration of the defects that occurred after bone resection. As shown in case 3, if further augmentation is needed, we can elevate an additional unipedicled flap from the contralateral side to a bipedicled one. Even in patients where the bone-plate fixation system cannot be removed, it would play the role of a buffer between the plate and the scalp on its superior part.

The skin defects occurring after the resection of the ulcer and the adjacent scar tissue can also be reconstructed with a skin graft following pericranial flap surgery. We attempted to reconstruct the defects using the primary suture, if possible, because alopecia that occurs at the recipient sites leads to poor aesthetic outcomes. In the current study, when we resected the ulcer, including the adjacent scar tissue, the size of the skin defects might have been greater than the ulcer and, moreover, the mobility of the flap might have been decreased because of the wide subgalea scarring formed by the repetitive surgical procedures. Therefore, we made 2-3 relaxation incisions, in parallel to the bilateral flap margin, at the galea aponeurotica, in order to increase the flap mobility [10]. In our experience, these relaxation incisions usually permit bilateral advancement of scalp flaps to primarily close scalp defects less $2 \mathrm{~cm}$ in width without tension. If the width of a scalp defect is over $2 \mathrm{~cm}$, we recommend skin grafting 


\section{Fig. 4. Various types of bipedicled pericranial flaps with} sufficient arterial blood supply

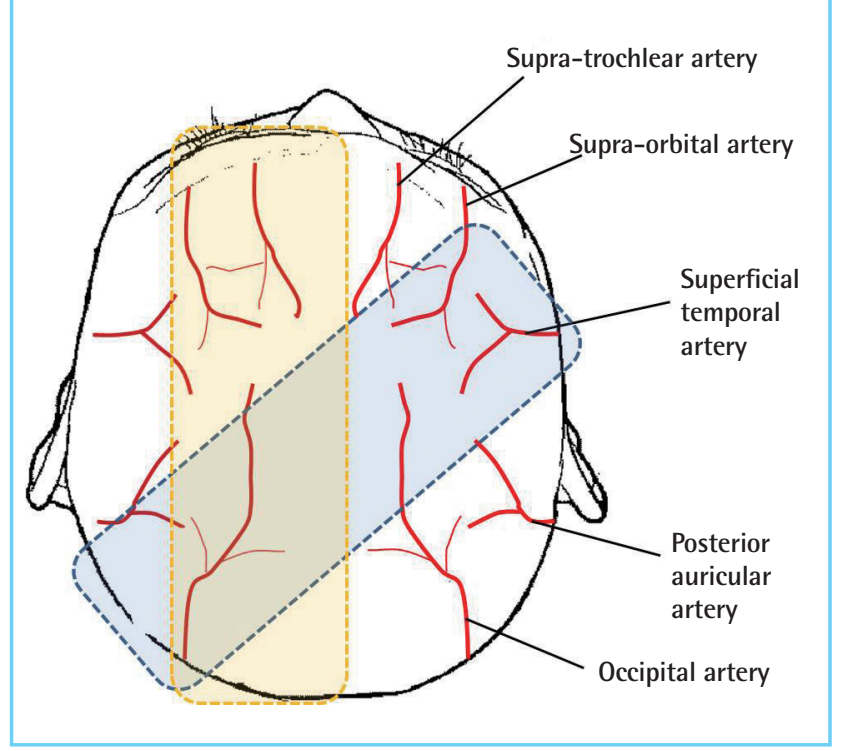

onto the pericranial flap or a large transposition or rotation flap. A transposition or rotation scalp flap should be designed on the normal scalp instead of on the previously elevated scalp, which would limit flap mobility because of severe subgalea scarring and previous incision scars.

\section{REFERENCES}

1. Argenta LC, Friedman RJ, Dingman RO, et al. The versatility of pericranial flaps. Plast Reconstr Surg 1985;76:695-702.

2. Al-Qattan MM. The use of multifolded pericranial flaps as "plugs" and "pads". Plast Reconstr Surg 2001;108:336-42.

3. Potparic Z, Fukuta K, Colen LB, et al. Galeo-pericranial flaps in the forehead: a study of blood supply and volumes. Br J Plast Surg 1996;49:519-28.

4. Boeckx WD, van der Hulst RR, Nanhekhan LV, et al. The role of free flaps in the treatment of persistent scalp osteomyelitis. Neurosurgery 2006;59:ONS64-7.

5. Leedy JE, Janis JE, Rohrich RJ. Reconstruction of acquired scalp defects: an algorithmic approach. Plast Reconstr Surg 2005; 116:54e-72e.

6. Lutz BS, Wei FC, Chen HC, et al. Reconstruction of scalp defects with free flaps in 30 cases. Br J Plast Surg 1998;51:18690.

7. Nakadai M, Takahashi H, Taniguchi M, et al. Repair of widely infected scalp defect and osteomyelitis with free radial forearm flap: a case report. No Shinkei Geka 1991;19:655-9.

8. Karsidag S, Ozcan A, Ozkaya O, et al. Use of wide bipedicled pericranial flap in anterior scalp reconstruction. J Craniofac Surg 2009;20:2248-51.

9. Tolhurst DE, Carstens MH, Greco RJ, et al. The surgical anatomy of the scalp. Plast Reconstr Surg 1991;87:603-12.

10. Burm JS, Oh SJ. Prevention and treatment of wide scar and alopecia in the scalp: wedge excision and double relaxation suture. Plast Reconstr Surg 1999;103:1143-9. 